\title{
Control of lupus nephritis by changes of gut microbiota
}

\author{
Qinghui Mu' ${ }^{1 \dagger}$, Husen Zhang ${ }^{2,7 \dagger}$, Xiaofeng Liao', Kaisen Lin², Hualan Liu' ${ }^{2,8}$, Michael R. Edwards', S. Ansar Ahmed', \\ Ruoxi Yuan ${ }^{1,3}$, Liwu Li ${ }^{3}$, Thomas E. Cecere ${ }^{1}$, David B. Branson ${ }^{1}$, Jay L. Kirby ${ }^{1}$, Poorna Goswami ${ }^{1}$, Caroline M. Leeth ${ }^{4}$, \\ Kaitlin A. Read ${ }^{5}$, Kenneth J. Oestreich ${ }^{5}$, Miranda D. Vieson ${ }^{1}$, Christopher M. Reilly ${ }^{1,6}$ and Xin M. Luo ${ }^{1 *}$
}

\begin{abstract}
Background: Systemic lupus erythematosus, characterized by persistent inflammation, is a complex autoimmune disorder with no known cure. Immunosuppressants used in treatment put patients at a higher risk of infections. New knowledge of disease modulators, such as symbiotic bacteria, can enable fine-tuning of parts of the immune system, rather than suppressing it altogether.

Results: Dysbiosis of gut microbiota promotes autoimmune disorders that damage extraintestinal organs. Here we report a role of gut microbiota in the pathogenesis of renal dysfunction in lupus. Using a classical model of lupus nephritis, MRL/Ipr, we found a marked depletion of Lactobacillales in the gut microbiota. Increasing Lactobacillales in the gut improved renal function of these mice and prolonged their survival. We used a mixture of 5 Lactobacillus strains (Lactobacillus oris, Lactobacillus rhamnosus, Lactobacillus reuteri, Lactobacillus johnsonii, and Lactobacillus gasseri), but L. reuteri and an uncultured Lactobacillus sp. accounted for most of the observed effects. Further studies revealed that MRL/Ipr mice possessed a "leaky" gut, which was reversed by increased Lactobacillus colonization. Lactobacillus treatment contributed to an anti-inflammatory environment by decreasing IL-6 and increasing IL-10 production in the gut. In the circulation, Lactobacillus treatment increased IL-10 and decreased IgG2a that is considered to be a major immune deposit in the kidney of MRL/Ipr mice. Inside the kidney, Lactobacillus treatment also skewed the Treg-Th17 balance towards a Treg phenotype. These beneficial effects were present in female and castrated male mice, but not in intact males, suggesting that the gut microbiota controls lupus nephritis in a sex hormone-dependent manner.
\end{abstract}

Conclusions: This work demonstrates essential mechanisms on how changes of the gut microbiota regulate lupusassociated immune responses in mice. Future studies are warranted to determine if these results can be replicated in human subjects.

Keywords: Gut microbiota, Lupus, Leaky gut, Autoimmunity

\section{Background}

Perturbation of gut microbiota is known to promote autoimmune disorders that include inflammatory bowel disease, type 1 diabetes, rheumatoid arthritis, and multiple sclerosis. However, little is known on the role of gut microbiota in systemic lupus erythematosus (SLE). SLE is a very complex autoimmune disorder with no known cure. It is characterized by severe and

\footnotetext{
*Correspondence: xinluo@vt.edu

${ }^{\dagger}$ Equal contributors

'Department of Biomedical Sciences and Pathobiology, Virginia-Maryland

College of Veterinary Medicine, Virginia Tech, Blacksburg, VA, USA

Full list of author information is available at the end of the article
}

persistent inflammation that damages multiple organs, including the skin, kidney, lung, joint, heart, and brain [1]. The prevalence ranges from 20 to 200 cases per 100,000 persons, with higher prevalence for people of African, Hispanic, or Asian ancestry. Although the disease affects both males and females, women of childbearing age are diagnosed 9 times more often than men. African-American women suffer from more severe symptoms and a higher mortality rate. More than half of SLE patients suffer from kidney inflammation, or lupus nephritis (LN), which is the leading cause of mortality by SLE. Current treatments for LN are primarily 
nonselective immunosuppressants [2]. While immunosuppression can effectively treat symptoms, unwanted side effects are a major cause of concern. Patients taking long-term immunosuppressants are prone to higher incidence of and more severe infections [3]. Therefore, there is an imperative need for new treatment strategies against LN. To accomplish this task, a better understanding of disease pathogenesis is required.

Current knowledge on the relationship between gut microbiota and SLE is limited [4]. In human SLE, a recent cross-sectional study showed dysregulated fecal microbiota of SLE individuals with a lower Firmicutes to Bacteroidetes ratio [5] that is consistent with gut dysbiosis observed in other autoimmune conditions [6, 7]. In mice, it has been reported that the lupus-prone MRL/ Mp-Fas ${ }^{l p r}(l p r)$ mouse model exhibits similar disease manifestations under specific pathogen-free and germfree conditions [8]. This suggests that complete removal of microbiota does not affect disease progression in these mice. The same phenomenon was observed in the pristane-induced lupus model [9]. However, completely depleting the microbiota might have neutralized the respective effects of "good" and "bad" microbes. Studies on germ-free New Zealand black mice showed mixed results, with less renal disease but more anti-nuclear antibodies [10-12]. Our research team has recently described the dynamics of fecal/colonic microbiota in $l p r$ mice that suggests a critical role of gut microbiota on lupus pathogenesis [13]. However, whether the change of gut microbiota is a driving force in SLE, or merely a result of disease status, remains unclear.

Here we show that intestinal permeability is increased in female lpr mice preceding the onset of kidney disease (i.e., a "leaky" gut) and that increasing gut colonization of Lactobacillales restores the mucosal barrier function and reduced kidney pathology. Such change in gut microbiota promotes an anti-inflammatory environment in the gut, suppressing expression of IL-6 in the mesenteric lymph node (MLN) while increasing the levels of IL-10 in circulation and periphery. In addition, the production and renal deposition of pathogenic IgG2a is repressed with increased Lactobacillales, suggesting a potential mechanism for the reduced kidney pathology. Moreover, we show that Lactobacillus spp. rebalances $\mathrm{T}$ cell subsets in the kidney, increasing regulatory $\mathrm{T}$ (Treg) cells and suppressing pathogenic T-helper (Th) 17 cells. This suggests another potential mechanism by which gut microbiota can modulate renal function. Interestingly, the effects of Lactobacillus spp. are only present in female and castrated male lpr mice, but not in intact males, indicating a role for sex hormones in the regulatory function of gut microbiota on lupus disease. Taken together, our results suggest that the presence of Lactobacillus spp. in the gut can attenuate kidney inflammation in lupus-prone mice in a sex hormonedependent manner.

\section{Results \\ Lactobacillus spp. attenuate LN}

When comparing the bacterial composition in the gut microbiota of lupus-prone $l p r$ mice vs. MRL control mice, we found that female lpr mice had a significantly lower abundance of Lactobacillales in the gut microbiota than MRL controls at 5 weeks of age and prior to the onset of lupus-like disease (Additional file 1: Figure $\mathrm{S} 1 \mathrm{~A})$. However, it was unclear whether the change was a cause or result of disease initiation. Therefore, we performed reciprocal cecal microbiota transplantation experiments from MRL to lpr mice (Additional file 1: Figure S1B) and vice versa. While the disease in MRL mice did not change after the transfer of cecal content from lpr mice (data not shown), MRL-to-lpr cecal transplantation led to significantly reduced production of autoantibodies against double-stranded (ds) DNA from the lower gastrointestinal tract (Additional file 1: Figure S1C). Since the gut microbiota of young MRL mice contained a higher abundance of Lactobacillales than lpr mice, we sought to determine if the decrease in disease could be due to the elevated Lactobacillales in $l p r$ mice that were transferred from MRL mice upon cecal transplantation. Indeed, lpr mice receiving MRL cecal content had more abundant Lactobacillales in the gut microbiota than untreated controls (Additional file 1: Figure S1D), suggesting a positive correlation between a higher abundance of gut-colonized Lactobacillales and improved lupus symptoms.

The bacterial order Lactobacillales includes Lactobacillus spp. that are known as beneficial bacteria. We thus examined the effect of these beneficial bacteria on $l p r$ mice by directly inoculating freshly cultured Lactobacillus isolates (Additional file 1: Figure S1E). We used a mixture of 5 Lactobacillus strains-Lactobacillus oris, Lactobacillus rhamnosus, Lactobacillus reuteri, Lactobacillus johnsonii, and Lactobacillus gasseri. Different Lactobacillus strains have been reported to exert different immunological functions $[14,15]$. Among the 5 strains, all except $L$. oris are known to colonize the gut. To improve engraftment of Lactobacillus spp., we pre-treated the mice with ampicillin, neomycin, vancomycin, and metronidazole for 2 days, followed by 2 days of resting to allow for excretion of the antibiotics prior to Lactobacillus treatment. The brief antibiotic treatment at the time of weaning did not change the disease severity (Additional file 1: Figures S1F and S1G). We found that weekly gavages of Lactobacillus spp. significantly increased the relative abundance of Lactobacillales in the gut microbiota at weeks 5 and 7 (Fig. 1a and Additional file 2: Table S1), significantly reduced the level of 


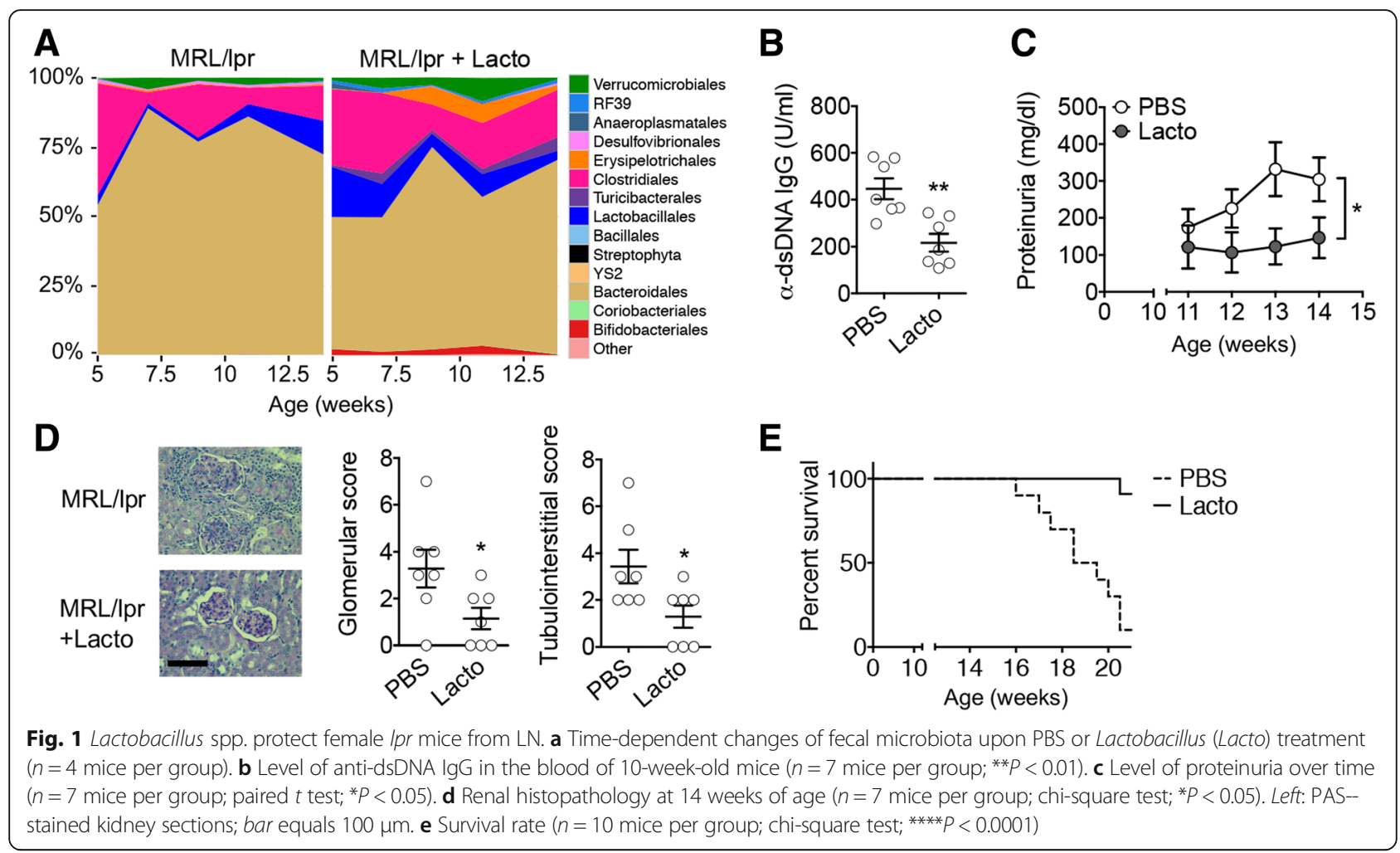

autoantibodies in the circulation (Fig. 1b), and significantly decreased proteinuria (Fig. 1c) and renal pathology scores (Fig. 1d). The spleen and MLN weights were not changed (Additional file 1: Figure S1H). Importantly, Lactobacillus treatment significantly increased the survival of female lpr mice (Fig. 1e). It is noteworthy that Lactobacillus treatment was given starting from 3 weeks of age and before disease establishment. When given after the onset of lupus disease, Lactobacillus treatment had a trend to reduce lupus disease, but the difference was not statistically significant (data not shown). These results suggest that the introduction of more "good" bacteria in the gut microbiota-in this case, Lactobacillus spp.-may be able to prevent disease progression in lupus-prone mice. This supports the notion that gut microbiota can directly control LN. How the increase of Lactobacilli in the gut affects disease pathogenesis in the kidney, which is extraintestinal, was unknown. Therefore, we next sought to identify potential "messengers" that transduced the disease-modulating signal from the gut to the kidney.

\section{A "leaky" gut in lupus-prone mice}

While 5 Lactobacillus strains were inoculated, we found by using $16 \mathrm{~S}$ ribosomal RNA gene sequencing that, unexpectedly, two bacterial species accounted for $>99 \%$ of the order Lactobacillales regardless of treatment status. The species were $L$. reuteri and an uncultured
Lactobacillus sp. (Fig. 2a). The same phenomenon was observed for MRL mice (data not shown). This suggests that $L$. reuteri and the uncultured Lactobacillus sp. accounted for most of the observed effects. As L. reuteri is known to enhance the epithelial barrier function of the gut $[16,17]$, we measured the level of endotoxin in the blood, and found it to be significantly higher in $l p r$ mice compared to the age-matched MRL controls (Fig. 2b). Interestingly, increasing colonization of Lactobacillales in the gut significantly decreased endotoxemia in lpr mice (Fig. 2c). These results suggest that the gut of lpr mice may be "leaky" and allow bacterial components (e.g., lipopolysaccharide, or LPS/endotoxin) to enter the blood stream. $L$. reuteri and the uncultured Lactobacillus sp., on the other hand, may be able to correct the leakiness. To test if the gut barrier was leaky in $l p r$ mice, we gavaged them with FITC-dextran and found significantly more FITC-dextran in the blood compared to MRL mice. When we treated the lpr mice with Lactobacillus spp., the levels of FITC-dextran in the circulation significantly decreased (Fig. 2d).

Two mucus layers cover the epithelial cells in the lower gastrointestinal tract [18]. Underneath the mucus layers, permeability of the intestinal epithelium is controlled by functions of tight junction proteins [19]. To determine whether $l p r$ mice had alterations in epithelial cell junctions, we isolated intestinal epithelial cells and measured the level of tight junction protein transcripts. 


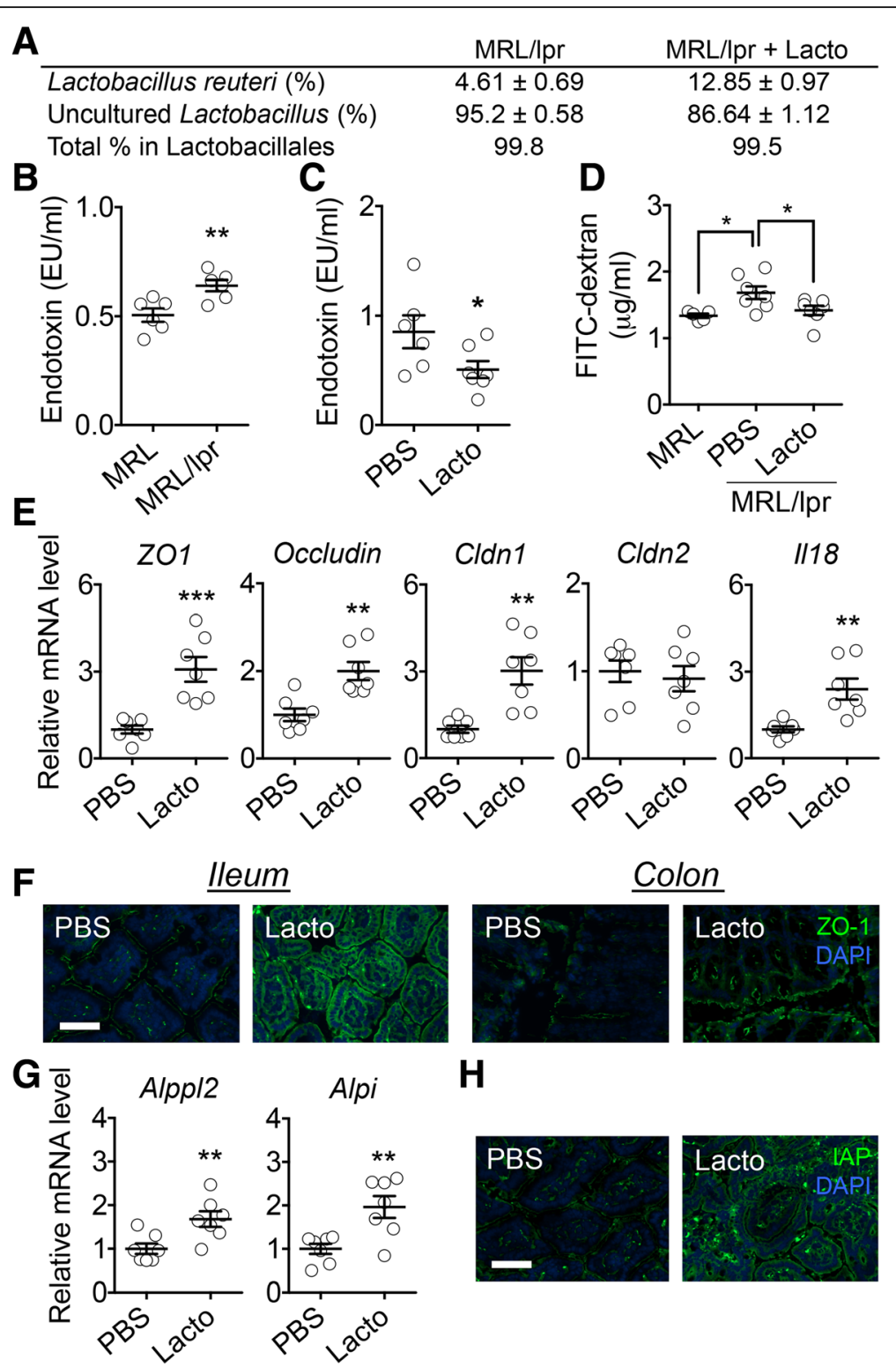

Fig. 2 Lactobacillus spp. restore gut mucosal barrier function in female Ipr mice. a Percentage of Lactobacillus strains in the order Lactobacillales ( $n=4$ per group). $\mathbf{b}$ Level of endotoxin in the blood of 6 -week-old lpr mice $\left(n=6\right.$ mice per group; $\left.{ }^{* *} p<0.01\right)$. $\mathbf{c}$ Level of endotoxin in the blood of 10-week-old Ipr mice with or without Lactobacillus treatment ( $n=6$ or 7 mice per group; ${ }^{*} P<0.05$ ). $\mathbf{d}$ Level of FITC-dextran diffused to the blood ( $n=5$ or 7 mice per group; ${ }^{*} P<0.05$ ). e Transcript levels of tight junction proteins and IL-18 in intestinal epithelial cells of 14-week-old Ipr mice $\left(n=7\right.$ mice per group; $\left.{ }^{* *} P<0.01,{ }^{* *} P<0.005\right)$. $\mathbf{f}$ Immunohistochemical stains of ZO-1 (green) in the ileum or colon. Nuclear stain (DAPI) is shown in blue. Bar equals $75 \mu \mathrm{m}$. $\mathbf{g}$ Transcript levels of IAP genes in the epithelium ( $n=7$ mice per group; $\left.{ }^{* *} P<0.01\right)$. $\mathbf{h}$ Immunohistochemical stains of IAP (green) in the ileum. Bar equals $75 \mu \mathrm{m}$

We found that treatment with Lactobacillus spp. significantly increased the expression of barrier-forming junction transcripts (ZO1, occludin, and Cldn1) without affecting the level of pore-forming junction transcript Cldn2 (Fig. 2e), suggesting enhanced barrier function of the intestinal epithelium with a higher abundance of Lactobacillales in the gut microbiota. Immunohistochemical analysis confirmed that the level of ZO-1 was increased by Lactobacillus treatment in both the ileum and the colon (Fig. 2f). We also found that epithelial expression of IL-18, a cytokine important for tissue repair [20] and limiting colonic T-helper 17 cell (Th17) differentiation [21], was significantly enhanced with Lactobacillus treatment (Fig. 2e). Interestingly, IL-18 can also be detrimental and promote inflammation in lpr mice [22]. We found that unlike epithelial expression, the level of IL-18 produced by MLN was significantly decreased by Lactobacillus treatment (data not shown). It is likely that 
Lactobacillus spp. can attenuate lupus disease through modulating the production of IL-18 from epithelial vs. immune cells.

In addition to strengthening intestinal mucosal barrier function, $L$. reuteri and the uncultured Lactobacillus sp. may also enhance LPS clearance by increasing the expression of intestinal alkaline phosphatase (IAP). IAP is a brush border enzyme expressed on the microvillus membranes of enterocytes [23] that can dephosphorylate LPS, leading to a 100-fold reduction in LPS toxicity [24]. In our studies, the epithelial expression of IAP (Alppl2 and Alpi) was significantly upregulated after Lactobacillus treatment in lpr mice compared to the controls (Fig. 2g). The upregulation of IAP was confirmed with immunohistochemical analysis (Fig. 2h). Interestingly, IAP has been reported to support the growth of Grampositive bacteria [25], which may explain the increase of Bifidobacteria in Lactobacillus-treated mice (Fig. 1a). Bifidobacteria can also promote gut epithelial integrity by strengthening tight junctions [26]. Together, these results suggest that gut microbiota can restore intestinal mucosal barrier function that is compromised in lupusprone lpr mice.

\section{Control of gut inflammation in lupus}

With an enhanced gut mucosal barrier, fewer bacteria are able to translocate across the intestinal epithelium leading to reduced activation and migration of CX3CR $1^{+}$ and/or $\mathrm{CD} 103^{+}$antigen-presenting cells (APCs) to the draining lymph nodes of the lower intestinal tract [27-29]. The decrease in APC migration may decrease the activation of $\mathrm{CD} 4^{ \pm} \mathrm{T}$ cells. Indeed, we found significantly decreased levels of $C x 3 \mathrm{cr} 1$ and Itgae (a subunit of CD103) specifically in the MLN with Lactobacillus treatment (Additional file 1: Figures S2A and S2B) suggesting that $L$. reuteri and the uncultured Lactobacillus sp. may reduce the migration of APC to the MLN. We next determined whether the activation of T cells was affected by the decrease of APC in the MLN. Upon activation, MLN T cells upregulate integrin $\alpha 4 \beta 7$ and chemokine receptor CCR9 for homing to the gut mucosa [30]. We found that Lactobacillus treatment significantly reduced the expression of both Itga4 and $C c r 9$ in the MLN (Additional file 1: Figures S2B and S2C), suggesting decreased activation of $\mathrm{T}$ cells. Consistent with this observation, migration of $\mathrm{T}$ cells to the intestinal lamina propria was reduced after mice were treated with the Lactobacillus spp. (Additional file 1: Figure S2D).

Among many pro-inflammatory cytokines produced by activated APC and T cells, IL-6 is known to promote antibody production from B cells [31] and suppress Treg cells [32], which are important for lupus progression in lpr mice [33-35]. We measured the transcript level of Il-6 in the MLN vs. spleen and found that it was significantly reduced by Lactobacillus treatment specifically in the MLN (Fig. 3a). CD4 ${ }^{+} \mathrm{CD} 8^{-} \mathrm{T}$ cells appeared to be a source of IL-6 in the MLN of lpr mice (Fig. 3b). As decreased IL- 6 would theoretically allow for differentiation of Treg cells [32], we next evaluated the levels of TGF $\beta$ and IL-10. Both cytokines were significantly increased at the transcriptional level in the MLN, but not the spleen, with Lactobacillus treatment (Fig. 3c), suggesting gutspecific immunosuppression. The serum TGF $\beta$ level was also significantly enhanced with the treatment (Fig. 3d), while the level of IL-6 in the circulation did not change (data not shown). Importantly, the induction of IL-10 with more Lactobacillales in the gut microbiota was not only in the MLN, but also systemic (Fig. 3e), suggesting that $L$. reuteri and the uncultured Lactobacillus sp. may exert a global anti-inflammatory function in $l p r$ mice through inducing IL-10 in the gut. Indeed, we also observed a significant elevation of IL-10 transcript levels in the kidney of $l p r$ mice with Lactobacillus treatment compared to untreated controls (Fig. 3f). Further analysis of MLN cells revealed that most IL-10-producing cells in the gut were $\mathrm{CD} 4^{+}$Foxp $3^{-}$type 1 regulatory $\mathrm{T}$ (Tr1) cells (Fig. 3g). This observation is consistent with published results on IL-10-producing Tr1 cells in $l p r$ mice [36]. Together, these results suggest that gut microbiota can promote an anti-inflammatory environment in the gut of lupus-prone mice, leading to induction of IL-10 that enters the circulation to provide systemic immunosuppression.

\section{Control of renal inflammation in lupus}

IL-10 can inhibit kidney disease in lpr mice through preventing IFN $\gamma$-mediated production of IgG2a, a major immune deposit in the kidney of these mice [37]. We found that Lactobacillus treatment significantly reduced the level of IgG2a in the blood (Fig. 4a) and its deposition in the kidney (Fig. 4b). This suggests that IgG2a may act as another "messenger" (in addition to IL-10) to transduce the disease-modulating signal from the gut to the kidney. The levels of IgG1 and total IgG did not change with the treatment (data not shown). Interestingly, the level of IgA was reduced by Lactobacillus treatment in the circulation (Fig. 4c), suggesting a potential effect of $L$. reuteri and the uncultured Lactobacillus sp. on class-switched antibodies. Indeed, the expression level of Aicda, whose gene product mediates class switch recombination [38], was significantly lower in the MLN of $l p r$ mice treated with Lactobacillus spp. (Fig. 4d). The change of IgA did not appear to be related to attenuation of LN, as it was not detectable in the kidney.

Different immune cell populations, including $\mathrm{T}, \mathrm{B}$, neutrophils, dendritic cells, and macrophages, have been demonstrated to infiltrate in the kidney with LN. To determine how Lactobacillus treatment affects immune cell migration to renal tissue, we evaluated various 


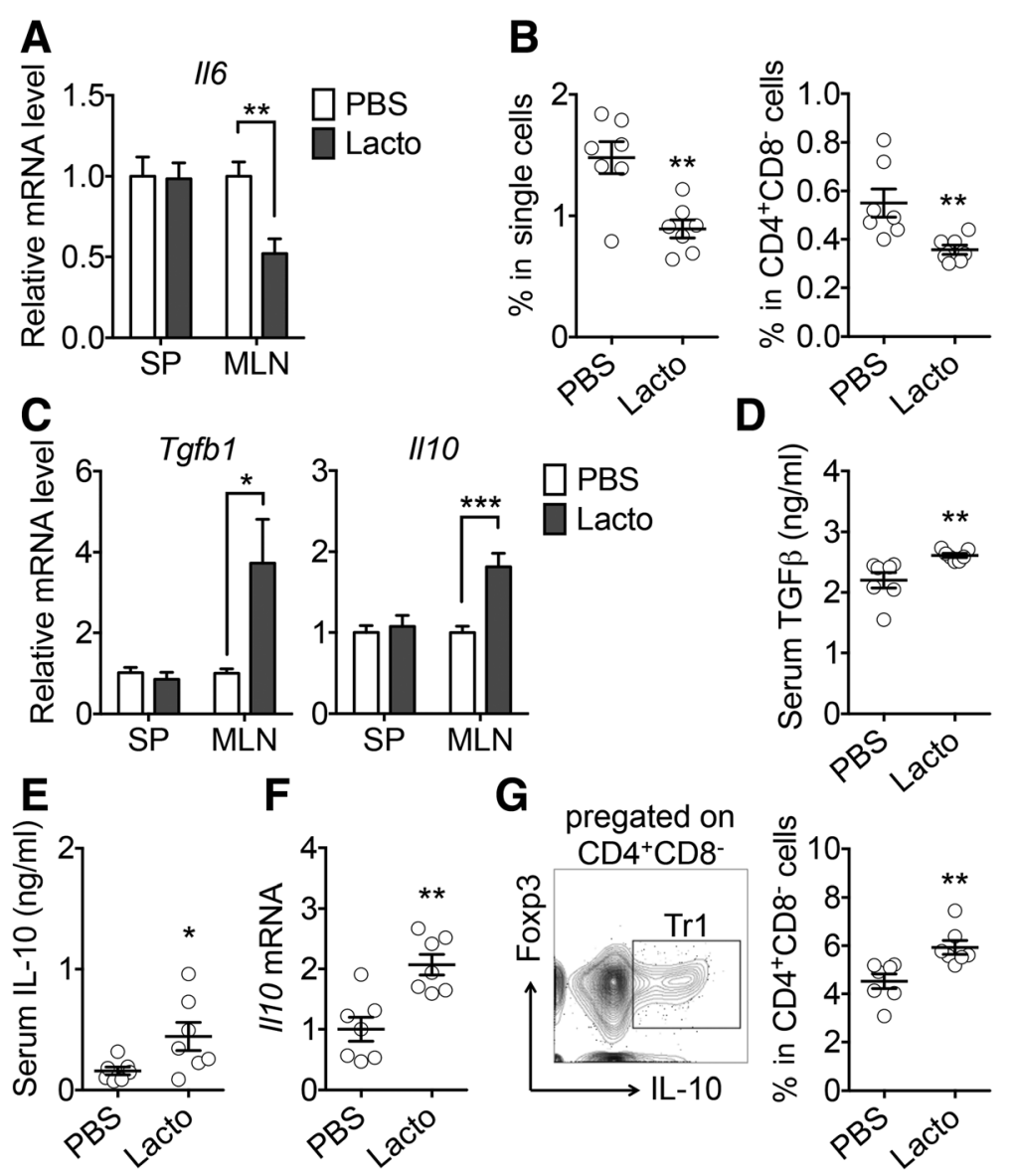

Fig. 3 Control of intestinal inflammation by gut microbiota in female Ipr mice. a Transcript level of IL-6 in the spleen (SP) and MLN ( $n=7$ mice per group; $\left.{ }^{* *} P<0.01\right)$. b Percentage of IL-6-expressing cells in the MLN $\left(n=7\right.$ mice per group; $\left.{ }^{* *} P<0.01\right)$. c Transcript levels of TGF $\beta$ and IL-10 $\left(n=7\right.$ mice per group; ${ }^{*} P<0.05$, $\left.{ }^{* * *} P<0.005\right)$. $\mathbf{d}$ Serum level of TGF $\beta\left(n=7\right.$ mice per group; $\left.{ }^{* *} P<0.01\right)$. e Serum level of IL-10 $(n=7$ mice per group; $\left.{ }^{*} P<0.05\right)$. f Transcript level of IL-10 in the kidney ( $n=7$ mice per group; $\left.{ }^{*} P<0.01\right)$. g FACS analysis of IL-10-expressing $\operatorname{Tr} 1$ cells in the MLN. Percentages of $\operatorname{Tr} 1$ cells in $C D 4^{+} C D 8^{-}$cells are shown $\left(n=7\right.$ mice per group; $\left.{ }^{* *} P<0.01\right)$

immune cell populations and found marked influx of $\mathrm{CD}^{+} \mathrm{T}$ cells, particularly $\mathrm{CD} 8^{+} \mathrm{T}$ cells, into the kidney of Lactobacillus-treated lpr mice (Fig. 4e). As $\mathrm{CD}^{+} \mathrm{T}$ cells are generally considered protective in lupus [39-41], it would suggest that renal infiltration of these cells exerts a suppressive effect on the development of LN. In addition, the number of Foxp $3^{+}$Treg cells significantly increased (Fig. 4f), while that of pathogenic Th17 cells significantly decreased (Fig. 4g), with Lactobacillus treatment. Together, these results suggest that gut microbiota may attenuate LN by limiting renal deposition of IgG2a and skewing the Treg-Th17 balance in the kidney towards Treg.

Sex hormones and gut microbiota cooperatively regulate $\mathrm{LN}$ SLE is a female-biased disease with women getting disease nearly 9:1 over men. The results shown so far were obtained from female mice. However, in lpr mice, both sexes get LN similarly. To investigate whether sex hormones and gut microbiota cooperatively regulate $\mathrm{LN}$ in $l p r$ mice, we treated male mice with the same Lactobacillus strains after mock or castration surgery (Additional file 1: Figure S3A). Bacterial profiling showed that Lactobacillus treatment increased the gut colonization of Lactobacillales in both mock and castrated mice (Additional file 1: Figure S3B and Additional file 3: Table S2). Strikingly, Lactobacillus treatment significantly decreased proteinuria (Fig. $5 \mathrm{a}$ ) and renal pathology (Fig. 5b) only in the castrated mice but not the intact animals, suggesting a possible role of androgenic hormones in suppressing the effects of Lactobacillus spp. The level of anti-double-stranded DNA (anti-dsDNA) IgG was not changed with Lactobacillus treatment (Additional file 1: Figure S3C). However, the total weight of lymph nodes (including mesenteric, renal, inguinal, lumbar, superficial, axillary/brachial, mediastinal lymph nodes) increased after mice were castrated, an effect reversed by Lactobacillus treatment (Additional file 1: Figure S3D). In 


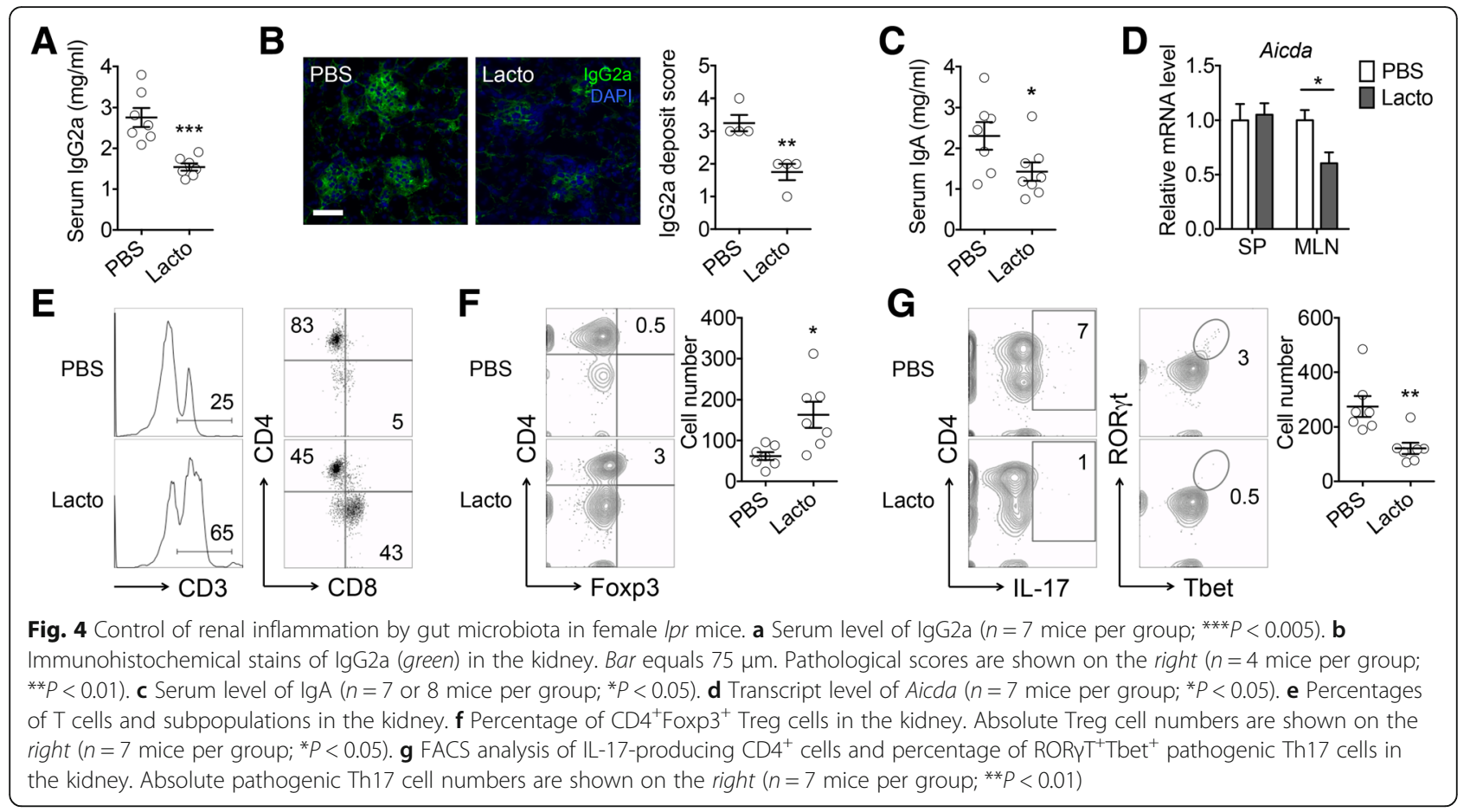

addition, increasing gut colonization of Lactobacillales significantly decreased the serum levels of IgG2a and IgA in castrated male mice, but not in the mice receiving mock surgery (Fig. 5c). The decrease of IgA appears to have originated from the colon (Additional file 1: Figure S3E), where the majority of Lactobacillus spp. (in terms of total number) resided [42]. Importantly, we found that unlike mice receiving in mock surgery, Lactobacillus treatment significantly increased the transcript levels of TGF $\beta$ and IL-10 in the MLN in castrated male lpr mice (Fig. 5d). Lactobacillus treatment also significantly increased circulating IL-10 in castrated animals only (Fig. 5e). Together, these results suggest that Lactobacillus treatment was not effective in intact male $l p r$ mice, while the response of castrated males to Lactobacillus treatment parallels that of female $l p r$ mice.

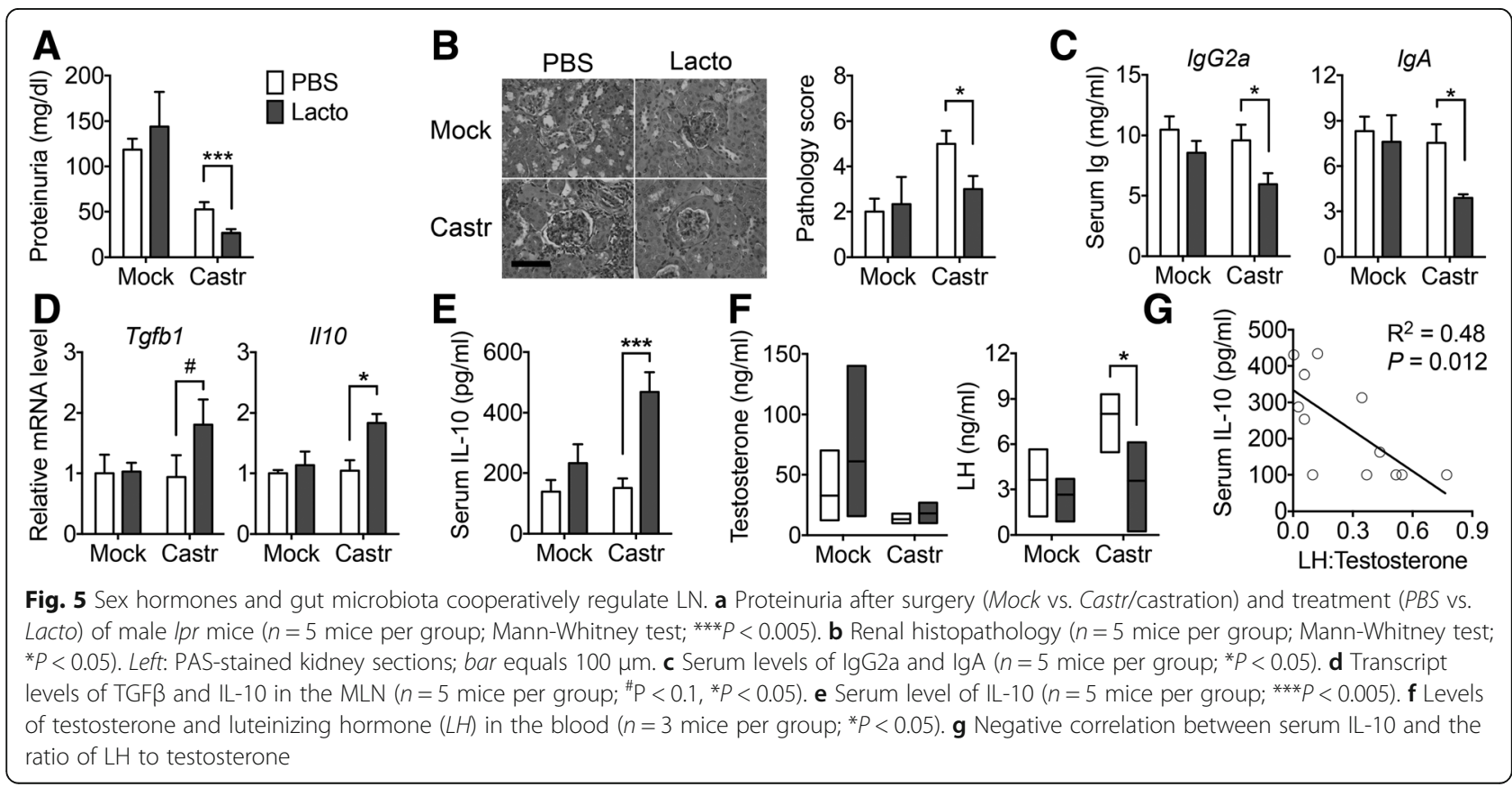


As testis is the only source of testosterone in mice, castration surgery completely removed the male hormone regardless of Lactobacillus treatment (Fig. 5f). We then measured two hormones regulated by testosterone, luteinizing hormone ( $\mathrm{LH})$, and follicle-stimulating hormone (FSH). Both are known to be repressed by testosterone [43-45]. As anticipated, castration surgery increased the levels of LH and FSH when the mice were not treated with Lactobacilli (Fig. 5f and Additional file 1: Figure S3F). However, Lactobacillus treatment significantly decreased the serum level of $\mathrm{LH}$, bringing it back to the level where testosterone was still present. We took the ratio of LH to testosterone and found it to be negatively correlated with serum IL-10 level (Fig. 5g). Whether LH directly affects IL-10, or vice versa, requires further investigation. Together, these results suggest that gut microbiota control $\mathrm{LN}$ in $l p r$ mice in a sex hormone-dependent manner. To determine the effect of Lactobacilli on sex differences, in future studies, we will transfer the cecal contents of young females to male mice to determine whether the interaction between sex hormones and Lactobacillus treatment is required for the observed changes in autoimmune response and/ or disease phenotype.

\section{Discussion}

The goal of this study was to understand the role of gut microbiota in the pathogenesis of SLE-associated kidney inflammation. In the $l p r$ model of LN, we found marked depletion of Lactobacillales in the gut microbiota compared to MRL controls. Increasing Lactobacillales in the gut microbiota improved the renal function of $l p r$ mice. Since Lactobacillus spp. are known to enhance the mucosal barrier function, the level of circulating endotoxin was measured. Endotoxin can accelerate nephritis in lupus-prone mice [46-48], and significantly higher endotoxemia was observed in $l p r$ mice preceding the onset of kidney disease. This suggests a "leaky gut" in pre-disease lpr mice. Lactobacillus treatment significantly decreased intestinal permeability in these mice and likely prevented detrimental bacteria and their antigens from penetrating the intestinal epithelium. Lactobacillus treatment also decreased CX3CR1 and CD103 expression in the MLN. CX3CR1- and CD103expressing cells are primarily APC $[29,49,50]$ that can capture bacteria from the gut lumen and transport them to the MLN, where they present antigens and activate $\mathrm{CD}^{+} \mathrm{T}$ cells to produce IL-6 that suppresses Treg, which is vital to lupus pathogenesis in $l p r$ mice. By preventing barrier compromise and decreasing microbial translocation, increased gut colonization of Lactobacillus spp. may reduce activation and migration of APC to the MLN, hence suppressing IL-6 production and allowing for Foxp $3^{-}$Tr1 cells to produce IL-10, which subsequently represses the synthesis and renal deposition of IgG2a. Inside the kidney, the Treg-Th17 balance was skewed towards Treg with Lactobacillus treatment. These effects of Lactobacilli, illustrated in Additional file 1: Figure S4, were absent in male mice unless castrated, suggesting that gut microbiota attenuates LN in a sex hormone-dependent manner. It is noteworthy that in these experiments, Lactobacillus treatment was given before disease establishment. It appears that $L$. reuteri and the uncultured Lactobacillus sp. have a preventative instead of curative effect on the development of LN.

Compromised intestinal barrier function has been reported in autoimmune conditions such as the inflammatory bowel disease (IBD), which includes Crohn's disease (CD) and ulcerative colitis (UC). It has been shown by using cecal biopsies that intestinal permeability is significantly increased in both CD and UC patients with irritable bowel syndrome-like symptoms than those with quiescent IBD without the symptoms [51]. This increase was accompanied by downregulation of the tight junction protein ZO-1. Endotoxemia in SLE patients that suggests disrupted gut mucosal barrier function in human SLE has also been reported [52]. In our studies, we show that the intestinal epithelium is compromised in lupus-prone $l p r$ mice and that Lactobacillus treatment can restore mucosal barrier function by increasing the expression of ZO-1. The effect of Lactobacilli on gut barrier function may also be attributed to the increase of Muc2, a mucin protein secreted by goblet cells that functions primarily to protect the intestinal epithelium [53].

The imbalance between anti-inflammatory Treg and inflammatory Th17 cells is widely recognized as being causative in the onset of both murine lupus and human SLE [54]. It is well established that environmental factors can promote plasticity between Treg and Th17 cells including the presence of inflammatory cytokines [55]. This cellular flexibility is due to effects of these inflammatory cytokines on the expression and function of the lineage-defining transcription factors Foxp3 and RORyt, which promote Treg and Th17 cell fates, respectively $[56,57]$. Intriguingly, changes in the composition of gut microbiota, particularly those of Clostridia and segmented filamentous bacteria (SFB) in mice and Bacteroides fragilis in humans, have been shown to alter the balance between Treg and Th17 cells [12]. We show here that increasing Lactobacillales in the gut microbiota can promote renal Treg cells and suppress diseasecausing Th17 cells to attenuate kidney inflammation in lupus-prone mice.

\section{Conclusions}

Environmental triggers initiate SLE in susceptible individuals. Since the gastrointestinal system serves as a first line of defense against various pathogens, delineating the 
type of flora and understanding the role the microbiota plays in determining disease susceptibility in SLE patients are paramount. We show in lupus-prone mice that Lactobacillus spp. in the gut microbiota exert antiinflammatory effects by repairing the damaged gut barrier, suppressing pro-inflammatory factors in the lymphatic circulation, and improving the ratio of regulatory versus pathogenic $\mathrm{T}$ cells, thereby attenuating kidney inflammation. While the relative abundance of Lactobacillales appears to be normal in SLE patients in remission (without active disease) [5], this does not preclude the possibility that beneficial bacteria capable of strengthening the gut barrier are lacking in SLE patients with active disease, especially those with LN. SLE is a very diverse disease; therefore, it is important to separately analyze the gut microbiota of SLE patients with different clinical manifestations. If the results of our mouse studies that L. reuteri and the uncultured Lactobacillus sp. which have a preventative effect on the development of LN can be replicated in humans, this may be a new avenue to identify at-risk individuals and provide protection in SLE-prone populations.

\section{Methods}

Mice

MRL/Mp (MRL), MRL/Mp-Fas ${ }^{l p r}$ (MRL/lpr or lpr, stock number 000485) mice were purchased from The Jackson Laboratory (Bar Harbor, ME) and bred and maintained in a specific-pathogen-free facility according to the requirements of the Institutional Animal Care and Use Committee at Virginia Polytechnic Institute and State university. Reciprocal cecal microbiota transplantation experiments were performed by diluting, under anaerobic conditions, contents of a cecum collected from one 3-week-old MRL or MRL/lpr donor mouse in $5 \mathrm{~mL}$ PBS. The cecal material was then suspended by vortexing, and the suspension was introduced by oral gavage into recipient mice at $0.2 \mathrm{~mL} /$ mouse when the mice were 3 weeks old and weaned. Another donor mouse was sacrificed on the next day, and the same procedure was repeated once. All Lactobacillus strains, including L. oris (F0423), L. rhamnosus (LMS201), L. reuteri (CF48-3A), L. johnsonii (135-1-CHN), and L. gasseri (JV-V03), were obtained from BEI Resources. All 5 strains were freshly cultured every week, mixed, and inoculated to MRL/lpr mice from 3 weeks old of age until dissection. For the experiment involving male castration, the testes and epididymis were removed through a scrotal incision under isoflurane inhalant anesthesia. The skin was closed using wound clips. Mock orchidectomy was performed on an equal number of mice to serve as surgical controls. The mock group of mice were prepared and anesthetized, and a scrotal incision was made; however, the incision was closed with a wound clip, without gonad removal.
All mice were administered ketoprofen, diluted to $0.5 \mathrm{mg} / \mathrm{mL}$ in sterile PBS, subcutaneously at $3.5 \mathrm{mg} / \mathrm{kg}$ as an analgesic post-operatively.

\section{Microbiota sampling and analysis}

Fecal microbiota samples were obtained by taking individual mice out of their cage and collecting a fecal pellet. To avoid cross-contamination, each microbiota sample was collected by using a new pair of sterile tweezers. Samples were stored at $-80{ }^{\circ} \mathrm{C}$ till being processed at the same time. Sample homogenization, cell lysis, and DNA extraction were performed as previously described [13]. PCR were performed, and purified amplicons were sequenced bidirectionally on an Illumina MiSeq at Argonne National Laboratory.

\section{Renal function}

Urine was collected biweekly, and all samples were stored at $-20{ }^{\circ} \mathrm{C}$ till being analyzed at the same time with a Pierce Coomassie Protein Assay Kit (Thermo Scientific). When the mice were euthanized at 14 weeks of age, the kidneys were fixed in formalin for $24 \mathrm{~h}$, paraffin embedded, sectioned, and stained with periodic acid-Schiff (PAS) at the Histopathology Laboratory at Virginia Maryland Regional College of Veterinary Medicine. Slides were read with an Olympus BX43 microscope. All the slides were scored in a blinded fashion by a certified veterinary pathologist. Glomerular lesions were graded on a scale of 0 to 3 for each of the following 5 categories: increased cellularity, increased mesangial matrix, necrosis, the percentage of sclerotic glomeruli, and the presence of crescents. Tubulointerstitial lesions were graded on a scale of 0 to 3 for each of the following four categories: presence of peritubular mononuclear infiltrates, tubular damage, interstitial fibrosis, and vasculitis.

\section{Endotoxin quantification and ELISA}

Separated serum after blood clotting was saved at $-20{ }^{\circ} \mathrm{C}$ until use. Serum endotoxin level was measured by using a Pierce LAL Chromogenic Endotoxin Quantitation Kit (Thermo Scientific). Anti-dsDNA IgG was measured according to a previously described method [58]. Serum IgG, IgA, IgG2a, and IL-10 concentrations were determined with mouse IgG, IgA, IgG2a (Bethyl Laboratories), and IL-10 (Biolegend) ELISA kits, respectively, according to the manufacturers' instructions.

\section{Immunohistochemistry}

The kidneys and 0.5-cm-length ileal and colonic sections were embedded in Tissue-Tek O.C.T. Compound (Sakura Finetek) and rapidly frozen in a freezing bath of dry ice and 2-methylbutane. Frozen OCT samples were cryosectioned and unstained slides were stored at $-80{ }^{\circ} \mathrm{C}$. Frozen 
slides were warmed to room temperature and let dry for $30 \mathrm{~min}$, followed by fixation in $-20{ }^{\circ} \mathrm{C}$ cold acetone at room temperature for $10 \mathrm{~min}$. After washing in PBS, the slides were blocked with PBS containing 1\% BSA for $20 \mathrm{~min}$ at room temperature. The slides were then incubated with fluorochrome-conjugated antibody mixture at room temperature in a dark humid box. The slides were mounted with Prolong Gold containing DAPI (Life Technologies). The following antibodies were used in immunohistochemical analysis: anti-mouse IgG2a-FITC (eBiosciense), rabbit anti-mouse $\mathrm{ZO}-1$ and FITCconjugated goat anti-rabbit IgG secondary antibody (Thermo Scientific), and rabbit anti-mouse IAP primary antibody (GeneTex). The slides were read and pictured with EVOS FL microscope (Advanced Microscopy Group) and $\mathrm{a} \times 20$ objective.

\section{Intestinal permeability}

In vivo intestinal permeability assay was performed by using FITC-conjugated dextran (Sigma-Aldrich). Briefly, mice were deprived of water overnight and then orally gavaged with FITC-dextran dissolved in PBS at $40 \mathrm{mg} / 100 \mathrm{~g}$ body weight (around $300 \mu \mathrm{L} / \mathrm{mouse}$ ). Mice were anesthetized after $4 \mathrm{~h}$, and the blood was collected and saved in the dark until serum separation. Serum was then diluted 1:1 with PBS and added to a 96-well microplate in duplicate, followed by determination of FITC concentration with Glomax (Promega) at an excitation of $485 \mathrm{~nm}$ and an emission wavelength of $528 \mathrm{~nm}$ using serially diluted FITC-dextran as the standard.

\section{Organ cultures}

Ileum and colon of $1 \mathrm{~cm}$ length were collected and opened longitudinally. Intestinal sections were thoroughly washed by PBS and cultured in 48-well plate with $500 \mu \mathrm{L} \mathrm{C} 10$ media at $37{ }^{\circ} \mathrm{C}$. Supernatant was collected after $24 \mathrm{~h}$ and analyzed by using ELISA.

\section{RT-quantitative PCR}

The spleen, MLN, and isolated intestinal epithelial cells (IECs; see below for isolation procedure) were homogenized with Bullet Blender homogenizer (Next Advance), and total RNA was extracted with RNeasy Plus Mini Kit (Qiagen) according to the manufacturers' instructions. Genomic DNA was removed by digestion with RNasefree DNase I (Qiagen). Reverse transcription was performed by using iScript cDNA Synthesis Kit (Bio-Rad). Quantitative PCR was performed with iTaq Universal SYBR Green Supermix (Bio-Rad) and ABI 7500 Fast Real-Time PCR System (Applied Biosystems). Relative quantities were calculated using L32 (MLN and spleen) and Villin (IECs) as the housekeeping gene. Primer sequences for mouse L32, Villin, ZO1, Occludin, Cldn1,
Cldn2, IL18, IL6, Tgfb1, IL10, Acida, CX3CR1, CCR9, Itgae, Itgb7, and Itga4 are available upon request.

\section{Cell isolation and flow cytometry}

The spleen, MLN, and Peyer's patches were collected and mashed in 70- $\mu \mathrm{m}$ cell strainers with C10 media. For splenocytes, red blood cells were lysed with RBC lysis buffer (eBioscience). To isolate lamina propria lymphocytes, the intestine was opened longitudinally and cut into pieces. The pieces were incubated twice in EDTA-DTT solution and intensively vortexed to remove the epithelial cell layer (saved as IEC-enriched fractions). After the second EDTA incubation, the pieces were cut and placed in a digestion solution containing $1 \mathrm{mg} / \mathrm{mL}$ collagenase $\mathrm{D}$ (Roche), $0.1 \mathrm{mg} / \mathrm{mL}$ DNase I (Sigma), and $10 \mu \mathrm{g} / \mathrm{mL}$ Dispase (Fisher). After digestion, the solution was passed through a $100-\mu \mathrm{m}$ cell strainer. The same process was repeated three times, and the supernatants of the three digestions were combined and added onto a 40:80 Percoll gradient to separate lymphocytes [59]. For surface marker staining, the cells were blocked by anti-mouse CD16/32 (eBioscience), stained with fluorochrome-conjugated antibodies, and analyzed with Attune NxT flow cytometer (Thermo Scientific). For intracellular staining, Foxp3 Fixation/Permeabilization kit (eBioscience) was used. Anti-mouse antibodies used in this study include the following: CD3-APC-eFluor 780, IL-6-FITC, CD8-PE-Cy7, Tbet-PerCP-Cy5.5, CD4-PerCP-Cy5.5, and ROR $\gamma$ T-PE (eBioscience); CD45-FITC, Foxp3-Alexa Fluor 647, IL-10BV421, and IL-17A-APC (Biolegend); and CD19-PerCPCy5.5, CD4-PE-Cy7, and CD8a-V450 (BD Biosciences). Flow cytometry data were analyzed with FlowJo.

\section{Hormone measurements}

Serum samples were saved at $-80{ }^{\circ} \mathrm{C}$ until analysis. Testosterone, luteinizing hormone, and follicle-stimulating hormone were measured at the University of Virginia Center for Research in Reproduction, which is supported by the Eunice Kennedy Shriver NICHD/NIH (NCTRI) Grant P50-HD28934.

\section{Statistical analysis}

For the comparison of two groups, unpaired Student's $t$ test was used unless specified. For the comparison of more than two groups, one-way ANOVA and Tukey's post-test were used. Results were considered statistically significant when $P<0.05 \quad\left({ }^{*} P<0.05,{ }^{* * *} P<0.01,{ }^{* * * *} P<\right.$ 0.005). All analyses were performed with Prism GraphPad.

\section{Additional files}

Additional file 1: Figure S1-S4. (A) Relative abundance of Lactobacillaceae in fecal microbiota ( $n=4$ per group; ${ }^{*} P<0.05$ at 5 weeks of age). (B) Study design of cecal transplantation from MRL to Ipr mice. (C) Level of 
anti-dsDNA IgG produced by 1-cm-long ileal or colonic organ culture after 24-h incubation $\left(n>3\right.$ per group; ${ }^{*}<<0.05$ ). (D) Time-dependent changes of fecal microbiota upon cecal transplantation. Abundant bacterial OTU $(>0.1 \%$ ) were summarized ( $n=4$ per group). (E) Study design of Lactobacillus treatment of Ipr mice. (F-G) Female MRL/lpr mice were treated with PBS control or mixed antibiotics (Abx) for 2 days at 3 weeks of age and sacrificed at 14 weeks of age ( $n=3$ per group). The levels of proteinuria (F) and antidsDNA antibodies (G) at 14 weeks of age are shown. The differences were not significant. (H) Weight of spleen and MLN of Ipr mice upon Lactobacillus treatment. Figure S2. (A) Transcript level of CX3CR1 in lymphoid tissues of Ipr mice treated with PBS or Lactobacilli. (B) Transcript levels of CD103 (Itgae and Itgb7) and a4b7 (Itga4 and ltgb7). (C) Transcript level of CCR9. (D) Percentage of CD3+ T cells in the intestinal lamina propria. ${ }^{*} P<0.05,{ }^{*} P<0.01$, ${ }^{* * *} P<0.001$. Figure S3. (A) Study design of surgery and treatment in male Ipr mice. (B) Time-dependent changes of fecal microbiota. Castr castration. (C) Level of anti-dsDNA lgG in the blood ( $n=5$ per group). (D) Total weight of lymph nodes (LN) from multiple sites $(* *<0.01)$. (E) Level of IgA produced by $1-\mathrm{cm}$ sections of ileal or colonic organ culture after 24 -h incubation $\left.{ }^{*} P<0.05\right)$. (F) Level of FSH in the blood. Figure S4. Working model (see text for details). (PDF $794 \mathrm{~kb}$ )

Additional file 2: Table S1. Actual abundance number and $P$ values for Fig. 1a. (PDF $30 \mathrm{~kb}$ )

Additional file 3: Table S2. Actual abundance numbers and $P$ values for Additional file 1: Figure S3B. (PDF $40 \mathrm{~kb}$ )

\section{Abbreviations}

CD: Crohn's disease; dsDNA: Double-stranded DNA; IAP: Intestinal alkaline phosphatase; IBD: Inflammatory bowel disease; IECs: Intestinal epithelial cells; LN: Lupus nephritis; Ipr: MRL/Ipr; LPS: Lipopolysaccharide; MLN: Mesenteric lymph node; PAS: Periodic acid-Schiff; SFB: Segmented filamentous bacteria; SLE: Systemic lupus erythematosus; Th17: T-helper 17 cells; Tr1: Type 1 regulatory T cells; Treg: Regulatory T cells; UC: Ulcerative colitis

\section{Acknowledgements}

We thank Sarah Owens for assistance on Illumina MiSeq sequencing, Melissa Makris for the use of flow cytometry core facility, and The University of Virginia Center for Research in Reproduction Ligand Assay and Analysis Core for the analysis of various hormones.

\section{Funding}

This work was supported by NIH R03-Al1 17597 and X.M.L.'s internal grants provided by Virginia Tech. The University of Virginia Center for Research in Reproduction Ligand Assay and Analysis Core is supported by the Eunice Kennedy Shriver NICHD/NIH (NCTRI) Grant P50-HD28934. The funding sources have no role in the design of the study and collection, analysis, and interpretation of the data or in writing the manuscript.

\section{Availability of data and materials}

The datasets generated and analyzed during the current study are available in the NCBI SRA accession number SRP078174.

\section{Authors' contributions}

$X M L$ designed the experiments. QM, XL, KL, HL, ME, RY, DB, JK, PG, and KR conducted the experiments. $\mathrm{HZ}$ analyzed the microbiome data. TC and MV scored the slides and evaluated kidney pathology. SA, LL, CL, CR, and KO assisted in experimental designs and contributed critical reagents. $\mathrm{XML}, \mathrm{QM}, \mathrm{HZ}, \mathrm{CR}$, and $\mathrm{KO}$ wrote the paper. All authors read and approved the final manuscript.

\section{Ethics approval}

This study was carried out in strict accordance with the recommendations in the Guide for the Care and Use of Laboratory Animals of the National Institutes of Health. The protocol was approved by the Institutional Animal Care and Use Committee (IACUC) of Virginia Tech College of Veterinary Medicine (Animal Welfare Assurance Number: A3208-01). For anesthesia and euthanasia, isoflurane and $\mathrm{CO} 2$ were used, respectively, according to the IACUC protocol.

\section{Consent for publication}

Not applicable.

\section{Competing interests}

The authors declare that they have no competing interests.

\section{Publisher's Note}

Springer Nature remains neutral with regard to jurisdictional claims in published maps and institutional affiliations.

\section{Author details}

${ }^{1}$ Department of Biomedical Sciences and Pathobiology, Virginia-Maryland College of Veterinary Medicine, Virginia Tech, Blacksburg, VA, USA. ${ }^{2}$ Department of Civil and Environmental Engineering, Virginia Tech, Blacksburg, VA, USA. ${ }^{3}$ Department of Biological Sciences, Virginia Tech, Blacksburg, VA, USA. ${ }^{4}$ Department of Animal and Poultry Sciences, Virginia Tech, Blacksburg, VA, USA. ${ }^{5}$ Virginia Tech Carilion Research Institute and School of Medicine, Roanoke, VA, USA. ${ }^{6}$ Edward Via College of Osteopathic Medicine, Blacksburg, VA, USA. ${ }^{7}$ Present Address: Cancer and Inflammation Program, Center for Cancer Research, National Cancer Institute, Bethesda, MD 20892, USA. ${ }^{8}$ Present Address: Lawrence Berkeley National Laboratory, Berkeley, CA 94720, USA.

Received: 21 October 2016 Accepted: 5 July 2017

Published online: 11 July 2017

\section{References}

1. Tsokos GC. Systemic lupus erythematosus. N Engl J Med. 2011;365(22):2110-21.

2. Lech $\mathrm{M}$, Anders HJ. The pathogenesis of lupus nephritis. J Am Soc Nephrol. 2013;24(9):1357-66.

3. Tektonidou MG, Wang Z, Dasgupta A, Ward MM. Burden of serious infections in adults with systemic lupus erythematosus: a national population-based study, 1996-2011. Arthritis Care Res (Hoboken). 2015;67(8):1078-85.

4. $\mathrm{Mu}$ Q, Zhang H, Luo XM. SLE: another autoimmune disorder influenced by microbes and diet? Front Immunol. 2015;6:608.

5. Hevia A, Milani C, Lopez P, Cuervo A, Arboleya S, Duranti S, et al. Intestinal dysbiosis associated with systemic lupus erythematosus. MBio. 2014;5(5): e01548-14.

6. Man SM, Kaakoush NO, Mitchell HM. The role of bacteria and patternrecognition receptors in Crohn's disease. Nat Rev Gastroenterol Hepatol. 2011;8(3):152-68.

7. Larsen N, Vogensen FK, van den Berg FW, Nielsen DS, Andreasen AS, Pedersen BK, et al. Gut microbiota in human adults with type 2 diabetes differs from non-diabetic adults. PLoS One. 2010;5(2):e9085.

8. Maldonado MA, Kakkanaiah V, MacDonald GC, Chen F, Reap EA, Balish E, et al. The role of environmental antigens in the spontaneous development of autoimmunity in MRL-Ipr mice. J Immunol. 1999;162(11):6322-30.

9. Mizutani A, Shaheen VM, Yoshida H, Akaogi J, Kuroda Y, Nacionales DC, et al. Pristane-induced autoimmunity in germ-free mice. Clin Immunol. 2005; 114(2):110-8.

10. East J, Prosser PR, Holborow EJ, Jaquet $H$. Autoimmune reactions and viruslike particles in germ-free NZB mice. Lancet. 1967;1 (7493):755-7.

11. Unni KK, Holley KE, McDuffie FC, Titus JL. Comparative study of NZB mice under germfree and conventional conditions. J Rheumatol. 1975;2(1):36-44.

12. Kosiewicz MM, Dryden GW, Chhabra A, Alard P. Relationship between gut microbiota and development of T cell associated disease. FEBS Lett. 2014;588(22):4195-206.

13. Zhang H, Liao X, Sparks JB, Luo XM. Dynamics of gut microbiota in autoimmune lupus. Appl Environ Microbiol. 2014;80(24):7551-60.

14. Lievin-Le Moal V, Servin AL. Anti-infective activities of lactobacillus strains in the human intestinal microbiota: from probiotics to gastrointestinal antiinfectious biotherapeutic agents. Clin Microbiol Rev. 2014:27(2):167-99.

15. Razmpoosh E, Javadi M, Ejtahed HS, Mirmiran P. Probiotics as beneficial agents in the management of diabetes mellitus: a systematic review. Diabetes Metab Res Rev. 2016;32(2):143-68.

16. Dicksved J, Schreiber O, Willing B, Petersson J, Rang S, Phillipson M, et al. Lactobacillus reuteri maintains a functional mucosal barrier during DSS treatment despite mucus layer dysfunction. PLoS One. 2012;7(9):e46399.

17. Liu F, Wen K, Li G, Yang X, Kocher J, Bui T, et al. Dual functions of Lactobacillus acidophilus NCFM as protection against rotavirus diarrhea. J Pediatr Gastroenterol Nutr. 2014;58(2):169-76.

18. Johansson ME, Phillipson M, Petersson J, Velcich A, Holm L, Hansson GC. The inner of the two Muc2 mucin-dependent mucus layers in colon is devoid of bacteria. Proc Natl Acad Sci U S A. 2008;105(39):15064-9. 
19. Suzuki T. Regulation of intestinal epithelial permeability by tight junctions. Cell Mol Life Sci. 2013;70(4):631-59.

20. Elinav E, Henao-Mejia J, Flavell RA. Integrative inflammasome activity in the regulation of intestinal mucosal immune responses. Mucosal Immunol. 2013;6(1):4-13

21. Harrison OJ, Srinivasan N, Pott J, Schiering C, Krausgruber T, llott NE, et al. Epithelial-derived IL-18 regulates Th17 cell differentiation and Foxp3(+) Treg cell function in the intestine. Mucosal Immunol. 2015;8(6):1226-36.

22. Schirmer B, Wedekind D, Glage S, Neumann D. Deletion of IL-18 expression ameliorates spontaneous kidney failure in MRLIpr mice. PLoS One. 2015;10(10):e0140173.

23. Geddes K, Philpott DJ. A new role for intestinal alkaline phosphatase in gut barrier maintenance. Gastroenterology. 2008;135(1):8-12.

24. Bates JM, Akerlund J, Mittge E, Guillemin K. Intestinal alkaline phosphatase detoxifies lipopolysaccharide and prevents inflammation in zebrafish in response to the gut microbiota. Cell Host Microbe. 2007;2(6):371-82.

25. Kaliannan K, Wang B, Li XY, Kim KJ, Kang JX. A host-microbiome interaction mediates the opposing effects of omega- 6 and omega-3 fatty acids on metabolic endotoxemia. Sci Rep. 2015;5:11276.

26. Hsieh CY, Osaka T, Moriyama E, Date Y, Kikuchi J, Tsuneda S. Strengthening of the intestinal epithelial tight junction by Bifidobacterium bifidum. Physiol Rep. 2015;3(3)

27. Farache J, Koren I, Milo I, Gurevich I, Kim KW, Zigmond E, et al. Luminal bacteria recruit CD103+ dendritic cells into the intestinal epithelium to sample bacterial antigens for presentation. Immunity. 2013;38(3):581-95.

28. Niess JH, Brand S, Gu X, Landsman L, Jung S, McCormick BA, et al. CX3CR1mediated dendritic cell access to the intestinal lumen and bacterial clearance. Science. 2005;307(5707):254-8.

29. Schulz O, Jaensson E, Persson EK, Liu X, Worbs T, Agace WW, et al. Intestinal CD103+, but not CX3CR1+, antigen sampling cells migrate in lymph and serve classical dendritic cell functions. J Exp Med. 2009;206(13):3101-14

30. Mora JR, von Andrian UH. Differentiation and homing of IgA-secreting cells. Mucosal Immunol. 2008;1(2):96-109.

31. Kishimoto T, Hirano T. Molecular regulation of B lymphocyte response. Annu Rev Immunol. 1988;6:485-512.

32. Kimura A, Kishimoto T. IL-6: regulator of Treg/Th17 balance. Eur J Immunol. 2010:40(7):1830-5.

33. Cash H, Relle M, Menke J, Brochhausen C, Jones SA, Topley N, et al. Interleukin $6(\mathrm{IL}-6)$ deficiency delays lupus nephritis in MRL-Faslpr mice: the IL-6 pathway as a new therapeutic target in treatment of autoimmune kidney disease in systemic lupus erythematosus. J Rheumatol. 2010;37(1):60-70.

34. Parietti $V$, Monneaux F, Decossas M, Muller S. Function of CD4+, CD25+ Treg cells in MRL/Ipr mice is compromised by intrinsic defects in antigen-presenting cells and effector T cells. Arthritis Rheum. 2008;58(6): 1751-61.

35. Shlomchik MJ, Madaio MP, Ni D, Trounstein M, Huszar D. The role of B cells in Ipr/lpr-induced autoimmunity. J Exp Med. 1994;180(4):1295-306.

36. Teichmann LL, Kashgarian M, Weaver CT, Roers A, Muller W, Shlomchik MJ. B cell-derived IL-10 does not regulate spontaneous systemic autoimmunity in MRL.Fas(lpr) mice. J Immunol. 2012;188(2):678-85.

37. Yin Z, Bahtiyar G, Zhang N, Liu L, Zhu P, Robert ME, et al. IL-10 regulates murine lupus. J Immunol. 2002;169(4):2148-55

38. Stavnezer J, Guikema JE, Schrader CE. Mechanism and regulation of class switch recombination. Annu Rev Immunol. 2008:26:261-92.

39. McPhee CG, Sproule TJ, Shin DM, Bubier JA, Schott WH, Steinbuck MP, et al. MHC class I family proteins retard systemic lupus erythematosus autoimmunity and B cell lymphomagenesis. J Immunol. 2011;187(9):4695-704.

40. Adachi $Y$, Inaba M, Sugihara A, Koshiji M, Sugiura K, Amoh $Y$, et al. Effects of administration of monoclonal antibodies (anti-CD4 or anti-CD8) on the development of autoimmune diseases in (NZW x BXSB)F1 mice. Immunobiology. 1998;198(4):451-64.

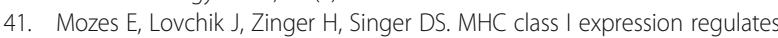
susceptibility to spontaneous autoimmune disease in (NZBXNZW)F1 mice. Lupus. 2005;14(4):308-14

42. Savage DC. Microbial ecology of the gastrointestinal tract. Annu Rev Microbiol. 1977;31:107-33.

43. Jean-Faucher $C$, el Watik N, Berger $M$, de Turckheim $M$, Veyssiere $G$ Jean $C$. Regulation of gonadotrophin secretion in male mice from birth to adulthood. Response to LRH injection, castration and testosterone replacement therapy. Acta Endocrinol (Copenh). 1985;110(2):193-9.
44. Shimizu H, Ohshima K, Bray GA, Peterson M, Swerdloff RS. Adrenalectomy and castration in the genetically obese (ob/ob) mouse. Obes Res. 1993;1(5):377-83.

45. Lindzey J, Wetsel WC, Couse JF, Stoker T, Cooper R, Korach KS. Effects of castration and chronic steroid treatments on hypothalamic gonadotropinreleasing hormone content and pituitary gonadotropins in male wild-type and estrogen receptor-alpha knockout mice. Endocrinology. 1998;139(10):4092-101.

46. Granholm NA, Cavallo T. Long-lasting effects of bacterial lipopolysaccharide promote progression of lupus nephritis in NZB/W mice. Lupus. 1994;3(6): 507-14.

47. Granholm NA, Cavallo T. Bacterial lipopolysaccharide enhances deposition of immune complexes and exacerbates nephritis in BXSB lupus-prone mice. Clin Exp Immunol. 1991;85(2):270-7.

48. Cavallo T, Granholm NA. Bacterial lipopolysaccharide transforms mesangial into proliferative lupus nephritis without interfering with processing of pathogenic immune complexes in NZB/W mice. Am J Pathol. 1990;137(4): 971-8.

49. Medina-Contreras $O$, Geem D, Laur O, Williams IR, Lira SA, Nusrat A, et al. CX3CR1 regulates intestinal macrophage homeostasis, bacterial translocation, and colitogenic Th17 responses in mice. J Clin Invest. 2011;121(12):4787-95

50. Diehl GE, Longman RS, Zhang JX, Breart B, Galan C, Cuesta A, et al. Microbiota restricts trafficking of bacteria to mesenteric lymph nodes by CX(3)CR1(hi) cells. Nature. 2013;494(7435):116-20.

51. Vivinus-Nebot M, Frin-Mathy G, Bzioueche H, Dainese R, Bernard G, Anty R, et al. Functional bowel symptoms in quiescent inflammatory bowel diseases: role of epithelial barrier disruption and low-grade inflammation. Gut. 2014;63(5):744-52.

52. Shi L, Zhang Z, Yu AM, Wang W, Wei Z, Akhter E, et al. The SLE transcriptome exhibits evidence of chronic endotoxin exposure and has widespread dysregulation of non-coding and coding RNAs. PLoS One. 2014;9(5):e93846.

53. Mack DR, Michail S, Wei S, McDougall L, Hollingsworth MA. Probiotics inhibit enteropathogenic E. coli adherence in vitro by inducing intestinal mucin gene expression. Am J Physiol. 1999;276(4 Pt 1):G941-50.

54. Alunno A, Bartoloni E, Bistoni O, Nocentini G, Ronchetti S, Caterbi S, et al. Balance between regulatory T and Th17 cells in systemic lupus erythematosus: the old and the new. Clin Dev Immunol. 2012;2012:823085.

55. Omenetti S, Pizarro TT. The Treg/Th17 axis: a dynamic balance regulated by the gut microbiome. Front Immunol. 2015;6:639.

56. Zhou L, Chong MM, Littman DR. Plasticity of CD4+ T cell lineage differentiation. Immunity. 2009;30(5):646-55.

57. Basu R, Hatton RD, Weaver CT. The Th17 family: flexibility follows function. Immunol Rev. 2013;252(1):89-103.

58. Liao X, Ren J, Wei CH, Ross AC, Cecere TE, Jortner BS, et al. Paradoxical effects of all-trans-retinoic acid on lupus-like disease in the MRL/lpr mouse model. PLoS One. 2015;10(3):e0118176.

59. Ivanov II, MCKenzie BS, Zhou L, Tadokoro CE, Lepelley A, Lafaille JJ, et al. The orphan nuclear receptor RORgammat directs the differentiation program of proinflammatory IL-17+ T helper cells. Cell. 2006;126(6):1121-33.

\section{Submit your next manuscript to BioMed Central and we will help you at every step:}

- We accept pre-submission inquiries

- Our selector tool helps you to find the most relevant journal

- We provide round the clock customer support

- Convenient online submission

- Thorough peer review

- Inclusion in PubMed and all major indexing services

- Maximum visibility for your research

Submit your manuscript at www.biomedcentral.com/submit 